\title{
Multiphysical Simulations Help to Ensure Assembled Printed Circuit Board and Power Components Reliability
}

\author{
Tomáš Sedláŕ ${ }^{1)}$ and Tibor Bachorec ${ }^{2)}$ \\ 1) SVS FEM s.r.o., Brno, Czech Republic, e-mail: tsedlar@svsfem.cz \\ 2) SVS FEM s.r.o., Brno, Czech Republic, e-mail: tbachorec@svsfem.cz
}

\begin{abstract}
Higher integration and reduced dimensions of components and printed circuit boards lead to rise of demands in precise design of electrical system. Numerical simulation enables designer to spot and repair errors in the electrical system without expensive prototyping and measuring. The paper summarizes possibilities of multiphysical analysis focused on problems with printed circuit boards and electrical power components with software ANSYS.
\end{abstract}

Keywords - PCB, IGBT, Joule heat, thermomechanical stress, numerical analysis, ANSYS.

\section{INTRODUCTION}

High reliability of printed circuit boards and mounted components is one of the key elements for high quality electronic asset. Reliability of electrical system on a printed circuit board or power elements can be influenced by thermal stress, that may cause not only the breakthrough of a semiconductor junction, but also deformation and possible break of trace due to thermal expansion. The most typical material for one- or two-layer PCB manufacturing is FR-4. The base material FR-4 is composed from glass fiber and epoxy resin. Therefore the physical parameters are orthotropic. Most of the multilayer circuit boards are made by lamination of prepreg with $\mathrm{Cu}$ foil on existing two-layer circuit board with FR-4 dielectric core. Figure 1 captures one of the possible fourlayer PCB setup. This is resulting in a "sandwich structure" of different materials that have different physical properties. Traces and paths are locally changing those material properties, especially thermal properties such as thermal conductivity and thermal expansion coefficient.

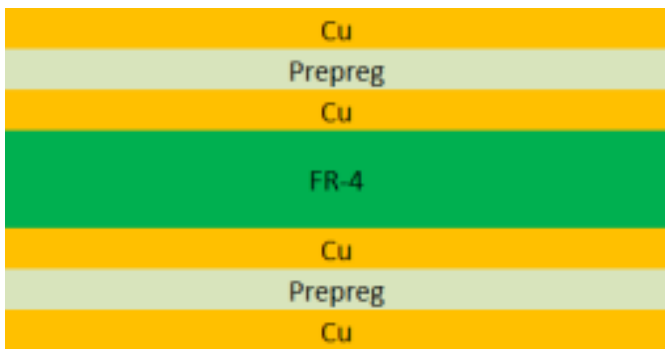

Fig. 1. Possible structure of 4-layer PCB.
Problems related to PCB and power components may be generally characterized as electrical, thermal and mechanical. Electrical problems involve signal and power integrity, cross induction, electromagnetic interference or ohmic losses in the system. Ohmic losses may lead to heating up the system and possible dysfunction, due to positive thermal feedback on semiconductor junction or due to thermomechanical stress. The most vulnerable to mechanical stress are solder joints, but also the trace or the contact lead can be broken due to this stress.

Electronic components or PCBs are mostly produced in big series. Therefore prevention of the mentioned faults during the system design is a must. Appropriate method for elimination of potential faults is numerical simulation during the development of the product. The portfolio of ANSYS tools are providing for calculation of any of these mentioned effects. The product can be simulated during normal or extreme working conditions. With interdisciplinary simulations ANSYS can cover effects from electrical, thermal and mechanical point of view for the system, so that every aspect is taken into account. Figure 2 shows interconnection possibilities among the ANSYS tools for the thermomechanical stress on PCB.

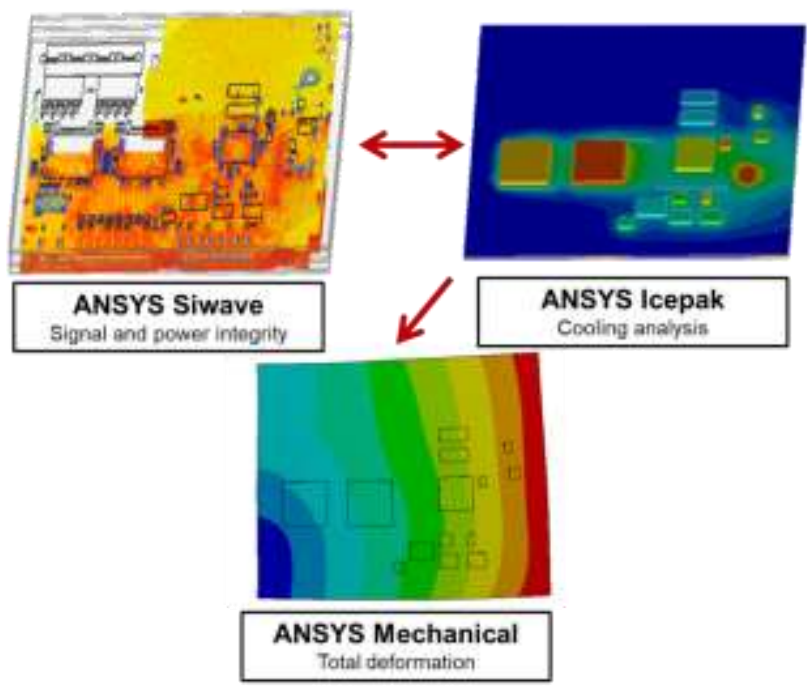

Fig. 2. Interconnection among different ANSYS tools during PCB simulation. 


\section{ANSYS SIWAVE}

ANSYS SIwave is specialized software for CPS (ChipPackage-System) simulation. Aside from basic DC analysis such as DCIR (Direct Current Internal Resistance) SIwave is providing for more complex analysis for power or signal integrity in time and frequency domain. Capabilities of SIwave designate it for problems in power electronics printed circuit boards and integrated circuits as well as for high frequency PCB systems. Typical applications are Joule heating simulation, identifying electromagnetic compatibility or interference in near or far field, cross-talk simulation, optimization of number and placement of filtering power rail capacitors, or extraction of RLCG parameters of traces.

Main advantages are adaptive meshing, import from various ECAD formats and automated iterative loop with ANSYS Icepak for electro-thermal analysis. Components on board can be described by IBIS model (Input/Output Buffer Information Specification). The setup of the electro-thermal analysis loop can be done in the SIwave GUI, where user can choose between natural or forced convection and its parameters, set the power dissipation of components, apply heatsinks on components and of course set up the convergence criteria for the coupling.

\section{ANSYS ICEPAK}

ANSYS Icepak is a tool for the thermal simulation for electronic applications. Main advantage is import of both MCAD and ECAD formats. Another perk is specialization on modelling of electronic components or printed circuit board, from coarse to highly detailed modelling. ANSYS Icepak leverages from ANSYS Fluent solver which is robust CFD finite volume method analyzer. ANSYS Icepak allows import of power maps from various tools, such as SIwave, Apache, Redhawk, Cadence and more. Trace mapping technology of ANSYS is able to perfectly recognize traces from dielectrics for local change in material properties. This technology is as precise as detailed MCAD modelling of traces, but saves the computing time and HW resources.

Figure 3 shows the difference between detailed explicit modelling and Trace mapping technique. On the left side we have object with ECAD data (Trace mapping). There are 7 objects in the model. On the other hand on the right side we have explicit model with MCAD data. There are 1581 objects, because the substrate layers and every trace are modelled as individual objects.
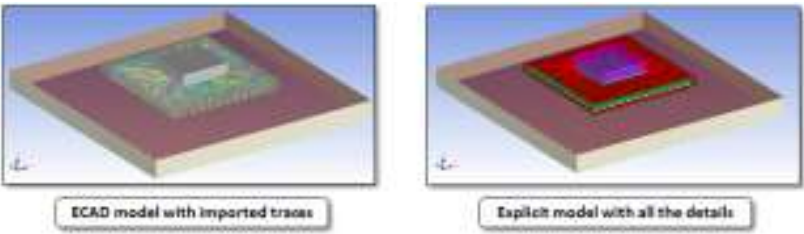

Fig. 3. Trace mapping vs explicit trace modelling.

Attitude with ECAD data has mesh with approximately 3.3 million elements against the MCAD design that has 11 million elements. Solve time is approximately more than five time faster in favor of the ECAD model. Table 1 summarizes the advantages of the Trace mapping against the explicit object modelling.
TABLE I.

COMPARISON OF MODELLING METHODS

\begin{tabular}{|c|c|c|c|c|}
\hline & $\begin{array}{c}\text { Number of } \\
\text { objects }\end{array}$ & $\begin{array}{c}\text { Mesh } \\
\text { Count }\end{array}$ & RAM & $\begin{array}{c}\text { Solve } \\
\text { Time }\end{array}$ \\
\hline $\begin{array}{c}\text { Trace } \\
\text { mapping }\end{array}$ & 7 & $\sim 3.3 \mathrm{M}$ & $0.24 \mathrm{X}$ & $0,18 \mathrm{X}$ \\
\hline $\begin{array}{c}\text { Explicit } \\
\text { modelling }\end{array}$ & 1581 & $\sim 11 \mathrm{M}$ & $1 \mathrm{X}$ & $1 \mathrm{X}$ \\
\hline
\end{tabular}

Typical application for ANSYS Icepak is cooling of an electronic system. Icepak can uncover if the heatsink or the fan or blower is sufficient for optimal thermal management. User can determine the optimal design with displayed contours and vectors of various variables, particle tracing or simple point values and table reports. Various parametrization and optimization that Icepak offers, also helps to reach the final design faster.

\section{ANSYS MECHANICAL}

ANSYS Mechanical is a worldwide known engineering tool for structural linear and nonlinear problems. ANSYS Mechanical is providing with solution for problems such as fatigue, various stresses, buckling stability, dynamic analysis: modal, harmonic, spectral, random excitation, transient, rotor dynamics, rigid body dynamics. Interaction among bodies is provided via contacts algorithms. As well as other ANSYS tools, ANSYS Mechanical can leverage from Trace mapping technique. Example of already done ECAD import to Mechanical module is in Fig. 4. ANSYS Mechanical can import temperature map from ANSYS Icepak to compute the thermomechanical stress. This stress can lead to deformation of substrate that can be easily visualized. With such results electrical engineer can adjust the whole design to maximize the reliability of the system.

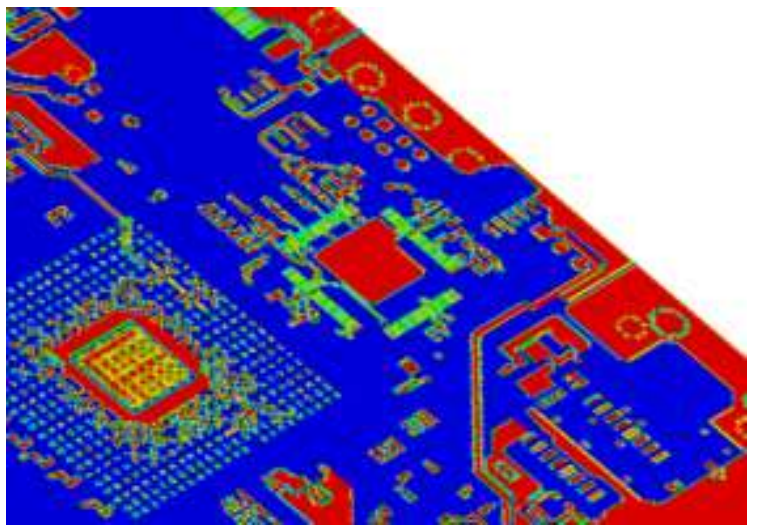

Fig. 4. ECAD import in ANSYS Mechanical.

\section{ANSYS TWINBUILDER}

ANSYS TwinBuilder is a system simulation tool. It offers various types of modelling such as electrical, mechanical, fluid models, state automats, block diagrams, algebraic and differential equations, models described in Modelica, VHDL-AMS, SML and $\mathrm{C} / \mathrm{C}++$. User can also import the ANSYS numerical finite element or finite volume model or chose to reduce its degree of freedom and import modelled application as a reduced order model (ROM). Such model drastically reduces computing demands and is solved in a "blink of an eye". Apart of fast ROM, user can connect simulation with models from Matlab, Mathcad, ModelSim, QuestaSim and SPICE. One 
of another unique features is creating power semiconductor device models of diodes, MOSFETs or IGBTs from datasheet or measurements for high fidelity simulations of switching, during which power loss on semiconductor junctions occurs. TwinBuilder can compute this power loss and use it as an excitation for thermal analysis from ANSYS Icepak. In this way user can obtain optimized thermal management for the system.

Figure 5 shows a segment of a system modeled in ANSYS TwinBuilder. Here the losses from IGBTs are connected to a heatsink model. The connections between IGBTs and pins of Icepak thermal model are bidirectional. For Icepak simulation they work as power excitation inputs with temperature outputs. Calculated temperatures are utilized in IGBT model for temperature dependent electrical properties and power loss output.

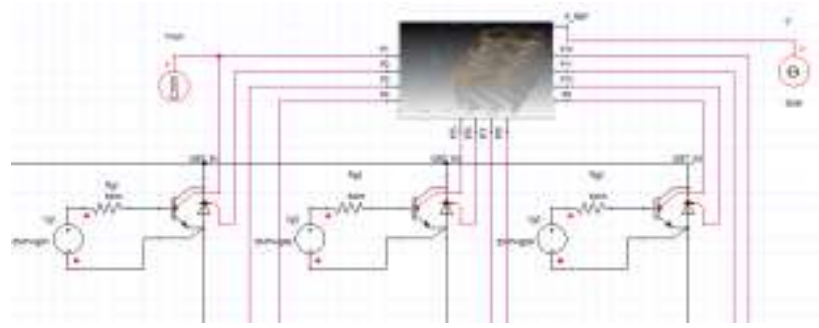

Fig. 5. IGBT models connected to thermal simulation in ANSYS TwinBuilder.
Newest feature of ANSYS TwinBuilder allows connecting simulation to an online sensor for two-way live communication. Sensor data can be fed into the system simulation as an excitation or data from the simulation can be sent to the sensor for controlling or driving the mechanism. This is a very useful for controlling our asset during its normal working conditions as we can provide the best maintenance or avoid failure states for irreparable systems.

\section{CONCLUSIONS}

ANSYS provides with whole portfolio of tools for coupled numerical analysis for electrical engineers. ANSYS Trace mapping can be used in electromagnetic, thermal and as well in mechanical type of simulation. This way engineer can leverage the advantage in whole simulation process. Analysis of the thermomechanical stress on PCB is faster and without major problems with convergence due to poor mesh quality or RAM capacity.

ANSYS TwinBuilder offers simulation of the whole system during the design phase from behavioral models to numerical ones as well as during the lifetime with data from asset's operating environment. The prediction for maintenance and failures has never been better before.

\section{REFERENCES}

[1] ANSYS Maxwell Technical Notes, ANSYS, Inc., 2018.

[2] ANSYS TwinBuilder Technical Notes, ANSYS, Inc., 2018.

[3] ANSYS Mechanical Technical Notes, ANSYS, Inc., 2018.

[4] ANSYS Icepak Technical Notes, ANSYS, Inc., 2018. 